University of Wollongong

Research Online

Faculty of Social Sciences - Papers (Archive) Faculty of Arts, Social Sciences \& Humanities

2015

Flourishing, languishing and moderate mental health: prevalence and change in mental health during recovery from drug and alcohol problems

Breanna McGaffin

University of Wollongong, bm425@uowmail.edu.au

Frank P. Deane

University of Wollongong, fdeane@uow.edu.au

Peter James Kelly

University of Wollongong, pkelly@uow.edu.au

Joseph Ciarrochi

Universty of Western Sydney, joec@uow.edu.au

Follow this and additional works at: https://ro.uow.edu.au/sspapers

Part of the Education Commons, and the Social and Behavioral Sciences Commons

Research Online is the open access institutional repository for the University of Wollongong. For further information contact the UOW Library: research-pubs@uow.edu.au 


\title{
Flourishing, languishing and moderate mental health: prevalence and change in mental health during recovery from drug and alcohol problems
}

\begin{abstract}
The aim was to explore the utility of Keyes' concept of mental health in a substance addiction context. Mental health is considered the presence of emotional wellbeing in conjunction with high levels of social and psychological functioning. Using Keyes' measure, the frequency of languishing and flourishing is compared between clients who became abstinent and those continuing to use substances following treatment. It was hypothesised that there would be a significant interaction between substance use and levels of mental health over time. Participants were 794 individuals (79.5\% male) attending residential substance abuse treatment provided by The Australian Salvation Army. The current sample was drawn from a larger longitudinal study evaluating routine client outcomes. At entry to treatment there were higher rates of languishing compared to population estimates, yet greater rates of flourishing at all time points compared to community normative data. There was a significant interaction between continuous mental health and substance use status. Mental health was rated significantly higher by individuals who were abstinent than those who had used substances at 3-month post-discharge follow-up. The comorbidity of mental illness and substance misuse has previously been investigated, but this is the first study to investigate the prevalence of mental health. While participants who remained abstinent achieved the highest levels of flourishing, at follow-up there were lower rates of languishing than found in a general community sample. Additionally, results suggested that improved mental health was a consequence of reduced severity of alcohol and other drug abuse, and followed reductions in cravings.
\end{abstract}

\section{Keywords}

alcohol, drug, recovery, during, change, problems, prevalence, flourishing, health, mental, moderate, languishing

\section{Disciplines}

Education | Social and Behavioral Sciences

\section{Publication Details}

McGaffin, B. Joy., Deane, F. P., Kelly, P. J. \& Ciarrochi, J. (2015). Flourishing, languishing and moderate mental health: prevalence and change in mental health during recovery from drug and alcohol problems. Addiction Research and Theory, 23 (5), 351-360. 


\section{ABSTRACT}

The aim was to explore the utility of Keyes' (2007) concept of mental health in a substance addiction context. Mental health is considered the presence of emotional wellbeing in conjunction with high levels of social and psychological functioning. Using Keyes' measure the frequency of languishing and flourishing is compared between clients who became abstinent and those continuing to use substances following treatment. It was hypothesised that there would be a significant interaction between substance use and levels of mental health over time. Participants were 794 individuals (79.5\% male) attending residential substance abuse treatment provided by The Australian Salvation Army. The current sample was drawn from a larger longitudinal study evaluating routine client outcomes. At entry to treatment there were higher rates of languishing compared to population estimates, yet greater rates of flourishing at all time points compared to community normative data. There was a significant interaction between continuous mental health and substance use status. Mental health was rated significantly higher by individuals who were abstinent than those that had used substances at 3-month post-discharge follow-up. The comorbidity of mental illness and substance misuse has previously been investigated but this is the first study to investigate the prevalence of mental health. While participants who remained abstinent achieved the highest levels of flourishing, at follow-up there were lower rates of languishing than found in a general community sample. Additionally, results suggested that improved mental health was a consequence of reduced severity of alcohol and other drug abuse, and followed reductions in cravings.

Keywords: flourishing; mental health; substance use disorders; recovery; recovery capital 


\section{INTRODUCTION}

"Recovery" is the aim of substance use disorder (SUD) treatment services (Laudet \& Humphreys, 2013). Historically abstinence has been one of the primary outcomes of recovery from SUDs (Garbutt, West, Carey, Lohr, \& Crews, 1999; Laudet \& White, 2010; Rudolf \& Watts, 2002). However, improvements in wider areas of functioning (such as wellbeing) can occur without abstaining (Laudet \& White, 2010). The Substance Abuse and Mental Health Services Administration (SAMHSA) recently defined recovery as "a process of change through which individuals improve their health and wellness, live a self-directed life and strive to reach their full potential” (SAMHSA, 2011). SAMHSA also identified four dimensions of life that promote recovery: health (physical and emotional, including abstinence), home (a safe residence), purpose (meaningful activity) and community (social network) (SAMHSA, 2011).

Consistent with this definition, research and treatment for many disorders are adopting wellness outcomes as indicators of recovery, predominantly with mental illness (e.g. schizophrenia, depression), and more recently substance misuse (Best et al., 2012; De Maeyer, Vanderplasschen, Lammertyn, van Nieuwenhuizen, \& Broekaert, 2011; Donovan, Mattson, Cisler, Longabaugh, \& Zweben, 2005). One such outcome is quality of life which captures elements of health and wellness (Donovan, et al., 2005), but more comprehensive components of subjective wellbeing are needed to operationalize definitions of recovery. A concept that has burgeoned in recent mental illness research is that of Keyes' model of complete mental health, which encompasses social, emotional, and psychological wellbeing (Keyes, 2007). Mental health is considered to be the presence of emotional wellbeing in conjunction with high levels of social and psychological functioning (Keyes \& Westerhof, 2012). 
Keyes' model of complete mental health has demonstrated that mental health and mental illness are independent yet correlated dimensions (Provencher \& Keyes, 2011). In this context, mental illness refers to psychopathology such as depression. In contrast the primary measure of mental health used to test the model of complete mental health, has been the Mental Health Continuum (Keyes, 2002) which can categorise or “diagnose” individuals as flourishing, languishing or moderately mentally healthy. To be flourishing in life, individuals must exhibit high levels of emotional wellbeing and positive functioning; in contrast a person who is languishing will exhibit low levels (Keyes, 2002). Individuals who do not meet the criteria for flourishing or languishing are considered moderately mentally healthy (Keyes, 2002). Flourishing, languishing and moderate mental health can all occur in the presence or absence of a mental illness (Keyes, 2002).

In relation to substance use, comorbidity of mental illness and addiction in Australian residential SUD clients has been reported at 64\%-71\% (Mortlock, Deane, \& Crowe, 2011). However, the prevalence of mental health has not been investigated in the context of substance misuse. Given the high prevalence of comorbidity, it has been proposed that addiction research should adopt and integrate constructs and outcomes utilised in the mental health recovery domain (Bowersox, Saunders, \& Wojcik, 2009; Coombs \& Meehan, 2003; Rudolf \& Watts, 2002). Given previous investigations of mental health and illness, we use substance use status as the measure of mental illness. If Keyes' (2002) conceptualisation of mental health is extended to the drug and alcohol addiction context, then it would be possible for individuals who were abstinent to potentially be flourishing, languishing, or moderately well. In addition to facilitating recovery progress, identifying mental health diagnoses would aid the mobilisation of additional treatment support to enhance recovery.

The interactions of well-functioning in the presence or absence of substance use is not new in the addiction fields. For example, languishing despite being abstinent has historically 
been captured by the concept of the "dry drunk". The phrase is a lay term, coined early in Alcoholics Anonymous history (AA; Flaherty, McGuire, \& Gatski, 1955) and is the notion that a person can be abstinent from substances yet still experience the emotional and functional problems that were encountered during their addiction (Gogek, 1994). It is theorised that there is a subset of individuals who, when discharged from treatment, are unable to attain satisfactory wellbeing, purpose in life, or flourish.

Keyes (2005) has reported American general population estimates of $16.9 \%$ of people who were languishing, $65.1 \%$ moderately mentally healthy and $18.0 \%$ considered to be flourishing from a mental health perspective. However, there appears to be variability across different populations. A survey study of 1,045 American yoga practitioners found notably different estimates, with $1.0 \%$ identified as languishing, $55.2 \%$ as moderately mentally healthy and 43.8\% as flourishing (Ross, Friedmann, Bevans, \& Thomas, 2013). The authors implied that yoga practice and belief in the personal health benefits of yoga might explain the relatively high rates of flourishing. However, definitive conclusions about why there were relatively high rates of flourishing in this sample could not be made from the cross-sectional data (Ross, et al., 2013).

Flourishing has been associated with a range of personal and societal health benefits (Keyes, 2005; Ross, et al., 2013). It has been found that individuals identified as flourishing have reduced odds of premature mortality, potentially due to the association of tobacco use and physical inactivity among individuals who are not flourishing (Keyes \& Simoes, 2012). When combined with the absence of a mental disorder, individuals who are flourishing have reported better health, such as the lowest risk of cardiovascular disease and fewest limitations of activities of daily living, and thus unsurprisingly have lower health care utilization and fewest missed days of work (Keyes, 2007). It is theorised that flourishing and moderate mental health are a source of resilience, acting as a buffer against stressful life events (Keyes, 
2002), which are known predictors of substance abuse relapse (Laudet, Cleland, Magura, Vogel, \& Knight, 2004). A potential mechanism for this buffer effect was identified by a survey study of community members across America (Catalino \& Fredrickson, 2011). They found that relative to individuals who did not flourish or were depressed, individuals who flourished tended to respond with larger spikes in positive emotion following everyday pleasant events. It was argued that this larger positive emotional reactivity ultimately fed back into promoting higher levels of flourishing. Therefore, the concept of flourishing may be a useful construct when considering outcomes following substance abuse treatment.

It is increasingly accepted that there is more to recovery than just abstinence from substances (Laudet \& White, 2010; Schwarzlose et al., 2007; Substance Abuse \& Mental Health Services Administration, 2011). There is a need to begin exploring the relationships between mental health as proposed by Keyes (2007) and substance use amongst individuals in treatment in order to identify those in need of greater support and more targeted interventions. Given the preliminary nature of this research on the mental health continuum, we have chosen to use both categorical and continuous ratings of mental health in order to provide comparisons with normative data and changes in mental health over time.

Therefore, the primary aim of the present study was to describe rates of mental health and to test the validity of the mental health continuum in the context of substance misuse. It was expected that mental health would have significant relationships with commonly utilised research and clinical measures, providing evidence of convergent and divergent validity. The second aim of the study was to identify variability in the proportions of categorical mental health “diagnoses” (Keyes, 2002). That is, to demonstrate whether individuals are, for example: abstinent and flourishing; misusing substances and languishing; misusing substances and flourishing; or abstinent and languishing. It is theorised that if recovery is broader than merely 'not drinking' then we should identify some individuals who are 
languishing despite being abstinent. The third aim of the research is to explore the temporal link between mental health and indices of substance abuse severity and cravings (alcohol use, drug use, and cravings). Three potential models are possible. The consequence model suggests that reduced addictive behaviour increases mental health. In short, given an episode of treatment it would be expected that substance abuse severity would decrease and this would lead to improvements in mental health. In contrast, the antecedent model assumes that poor mental health is the cause of poor substance use outcomes. There are several possible reasons why an antecedent model might be present. People who have poorer mental health may turn to the substances as an unhelpful way of coping. Such a model is consistent with the self-medication hypothesis and findings that some individuals use drugs and alcohol as a way to regulate positive and negative emotions (Cooper, Frone, Russell, \& Mudar, 1995;

Khantzian, 1997). In a treatment context it is also possible that poorer mental health makes it more difficult for those receiving drug and alcohol services to obtain improvements for their addictions. Finally, the reciprocal influence model suggests that substance use is both an antecedent to and a consequence of mental health problems.

\section{Method}

All measures, forms and procedures were approved by the University Human Research Ethics Committee.

\section{Participants}

The data for the current study was collected as part of a wider research initiative that involved evaluating the effectiveness of The Salvation Army Recovery Service Centres. These centres provide residential alcohol and other substance abuse treatment in the form of a modified therapeutic community. Participants were recruited from nine different Recovery Service Centres that were located in the Australian states of New South Wales, Queensland, 
and the Australian Capital Territory. Only participants who provided complete responses at entry to the program on the Mental Health Continuum (Keyes, 2002) and items indicating their prior 30-day substance use status (used or abstained) were included in the current analysis $(\mathrm{N}=794)$. Figure 1 provides a flow-chart of participant movement over the study period. The sample included 631 males (79.5\%) and 163 females, who had an average age of $36.09(S D=10.72)$. Participant demographic information is reported in Table 1 .

A follow-up rate of $28.8 \%$ was obtained at the 3-month follow-up; three participants did not provide complete responses to the mental health or use status items, resulting in a sample of 226 individuals. Only those participants contacted at the 3-month follow-up were re-contacted at 12-month follow-up. This resulted in 113 (50.9\%) participants providing complete responses to mental health and use status items.

[INSERT TABLE 1]

[INSERT FIGURE 1]

\section{Measures}

Mental Health Continuum - Short Form. The Mental Health Continuum - Short Form (MHC-SF) is a 14 item self-report questionnaire that assesses positive mental health (Keyes, 2002). Participants rate the frequency of each feeling in the past month on a 6 point Likert scale $(0=$ never to 5 = every day). It includes, 3 items form the Emotional Wellbeing subscale which assesses positive emotions towards one’s life ("Satisfied with life”), 5 items form the Social Wellbeing subscale ("That you had something important to contribute to society") and six items form the Psychological Wellbeing subscale ("That you liked most parts of your personality”) which assesses engagement and functioning in one’s social and private life. Cronbach's alpha in the current sample was satisfactory $(\alpha=.94)$.

The scale can be scored both continuously and categorically (Keyes, 2009). Continuous scoring is the sum of responses to the 14 items, with higher scores indicating better mental 
health. Categorical scoring results in what Keyes refers to as "diagnoses" of flourishing, languishing or moderate mental health (Keyes, 2002). To be flourishing, individuals must respond 'every day' or 'almost every day' to at least one of the three emotional wellbeing items, and at least six of the 11 social and psychological wellbeing items. To be languishing, individuals will respond 'never' or 'once or twice' to at least one emotional wellbeing item and six social and psychological wellbeing items. Individuals who are neither flourishing nor languishing are diagnosed with moderate mental health. Both scoring methods are utilised in the current research.

Addiction Severity Index. The Addiction Severity Index (ASI) is a semi-structured interview commonly used as a component of comprehensive assessment in substance abuse treatment programs (McLellan, Kushner, Metzger, Peters, \& et al., 1992). Several domains are assessed; drug, alcohol, medical, family, psychiatric, employment, and legal. Cronbach alpha's are acceptable for the composites ( $\alpha=.67$ to .85$)$ (Zanis, McLellan, \& Corse, 1997). For the purpose of the current research, only the questions pertaining to use of substances in the past 30 days were included in the analyses.

Life Engagement Test. The Life Engagement Test (LET) is a 6-item scale measuring a person's purpose in life in terms of engaging in activities that are personally valued (Scheier et al., 2006). Participants were asked to rate each statement on a scale from 1 (strongly disagree) to 5 (strongly agree). An example item is, “To me, the things I do are worthwhile”. Cronbach's alpha for the scale in the current sample was satisfactory $(\alpha=.77)$.

Depression Anxiety Stress Scale. The Depression Anxiety Stress Scale (DASS) is a 21 item self-report measure yielding three subscales (Lovibond \& Lovibond, 1995). Seven items form each of the subscales; depression ("I felt that life was meaningless"), anxiety ("I was aware of dryness of my mouth”), and stress (“I find it hard to wind down”). Cronbach’s alpha for the scale in the current sample is satisfactory $(\alpha=.96)$. 
Desires for Alcohol Questionnaire. The abbreviated 6 item Desires for Alcohol Questionnaire (DAQ) was used to assess the participants current desire for alcohol (Mo, Deane, Lyons, \& Kelly, 2013). Participants indicated their agreement to the statements on a 7 point Likert scale $(1=$ strongly disagree to $7=$ strongly agree). Items were modified for the study to assess drug and alcohol desires. For example, the statement "I want to drink so much I can taste it” was adjusted to "I want to drink/use drugs so much I can taste it”. Cronbach’s alpha for the scale in the current sample was satisfactory $(\alpha=.93)$.

Drug Taking Confidence Questionnaire. The Drug Taking Confidence Questionnaire (DTCQ) is an 8 item scale assessing a person's self-efficacy to resist the urge to drink alcohol or take drugs in specific high relapse risk situations (Sklar \& Turner, 1999). Participants were asked to specify their primary drug of choice then rate their confidence of resisting that drug in each situation on a scale of 0 (not at all confident) to 100 (very confident). This measure was only administered at baseline. Cronbach's alpha for the scale in the current sample was satisfactory $(\alpha=.91)$.

\section{Procedures}

The Salvation Army staff (centre managers and clinical employees) were trained in the administration of the ASI and all outcome measures used in the study by the research team. These measures were integrated into intake protocols and each client was provided a consent form and information relating to the aims, procedures and demands of the research. Those clients wishing to participate completed all measures during this intake session. Intake data was entered by The Salvation Army staff into the Salvation Army's online Service and Mission Information System (SAMIS) and downloaded for analysis by the research team.

Three and 12 month post-discharge follow-ups were conducted. Participants were provided with an AUD\$20 gift voucher for completing each follow-up interview (See Deane, Kelly, Crowe, Lyons, \& Cridland, 2014 for more details). 


\section{Data Analytic Strategy}

Visual inspections of the variables’ distributions (Tabachnick \& Fidell, 2013) showed normality violations, which transformations failed to correct, so non-parametric equivalents were adopted as required.

Spearman's Rho bivariate correlations were used to determine the associations between mental health and other routine clinical measures of SUDs. To determine proportional differences in categorical diagnoses (flourishing, languishing, moderate mental health) at each assessment, a Friedman two-way ANOVA was run. A Wilcoxon Signed Rank test was subsequently utilised to identify the assessment points that yielded significantly different diagnoses. To investigate the relationship between substance use status and mental health diagnosis, a Pearson’s Chi-square Test of Contingencies was used.

A 2 (Group: abstinent or used) x 3 (Time: baseline, 3-month, 12-month follow-up) mixed-design ANOVA and Autoregressive Cross-lag (ACL) analysis was used to examine changes in mental health across time for substance use status. With respect to the ANOVA, mental health scores obtained at the three time points were entered as a within-subjects factor and substance use at 3-month follow-up (abstinent and use of substances) was entered as a between subjects factor.

ACL models were utilised, as they are a common method to consider temporal ordering of constructs in order to distinguish between alternative causal hypotheses, or directionality of the associations between constructs (i.e., a predicts changes in b; b predicts changes in a; or a and b are reciprocally related (Marshall, Parker, Ciarrochi, \& Heaven, 2014). This model's focus is on the relations between one construct at a time point $T$ on change in another construct observed to occur between time point $\mathrm{T}$ and $\mathrm{T}+1$.

We used AMOS 21 (Arbuckle, 2012) to estimate a series of structural equation models representing the relations between substance use and mental health across the three 
time points of the study. We estimated one and two year autocorrelations (Time 1 predicting the same variable at Time 2 and 3) and one year cross-lags (Time 1 predicting the other Time 2 variable). Adding a two-year cross-lag did not improve fits of any model.

Given that this was a longitudinal study, missing data is a potential concern. It is now well recognized in the social sciences that traditional approaches to missing data (e.g., listwise or pairwise deletion) are inappropriate and can lead to biased parameter estimates. Modern methods like full-information-maximum-likelihood (FIML) provide a principled approach to missing data which uses all the available information for parameter estimation (Enders \& Bandalos, 2001; Howell, 2008). This procedure was employed for all models.

\section{Results}

\section{Attrition Bias}

The low follow-up rates have meant that there is considerable missing data. A more extensive missing data analysis for the follow-up methods used as part of routine outcome assessment revealed no systematic differences between completers and non-completers (Deane, et al., 2014). However, we also checked for potential attrition bias on 18 variables for the current subsample.

Differences between participants who had provided data at all three time points and those who had only completed the baseline assessment were investigated. An independent ttest of baseline variables (for example, religiosity, addiction severity) identified no significant differences, with the exception of the ASI alcohol composite score $(t(740)=-2.36, p<.05)$, and age $(t(794)=-3.72, p<.05)$. Participants who had not completed all follow-up assessments had lower alcohol severity and were younger $(M=35.53, S D=10.43)$ than participants who had completed assessments at all three time points $(M=39.58, S D=11.80)$. 
The following results are therefore more applicable to those clients with more severe alcohol problems and near our obtained mean age.

The impact of length in residential care was also considered. The analyses were conducted excluding individuals who had been at the treatment facility for less than one month. However, the same pattern of results was obtained. Given the additional data loss this introduced, and no significant difference identified in the independent t-test, time in treatment was not used an exclusion criterion.

\section{Correlations with Clinical Measures}

Spearman's Rho correlations were run due to the normality violations to assess the bivariate associations between the mental health continuum and the four self-report measures at baseline, 3-month follow-up and 12-month follow-up (Table 2). The correlations demonstrate moderate significant relationships in the expected direction. Mental health was negatively correlated with psychological symptom distress and cravings. Mental health was positively correlated with refusal self-confidence and life engagement.

\section{[INSERT TABLE 2 HERE]}

\section{Categorical Mental Health Prevalence}

Table 3 presents the frequencies of mental health as diagnosed categorically. All three diagnoses are present in the sample. Most participants were moderately mentally healthy at all three assessments (54.3\%, 50.0\%, and 47.8\% respectively). At baseline around as many participants were flourishing (21.9\%) as were languishing (23.8\%). However, at 3- and 12month follow-ups flourishing increased, whereas languishing decreased, when compared to baseline.

[INSERT TABLE 3 HERE] 


\section{Comparisons over Time}

A Friedman two-way ANOVA was used for participants who had completed the baseline, 3-month, and 12-month assessments to investigate differences in diagnoses over time. The results indicated that there was a statistical difference in the categorical mental health continuum scores at each assessment, $\chi^{2}(2, N=111)=24.33, p<.001$. Follow-up pairwise comparisons with the Wilcoxon Signed Rank test and a Bonferroni adjusted $\alpha$ of .017 indicated that there were significant differences between baseline (Mean Rank $=1.71$ ) and 3-month follow-up (Mean Rank = 2.18), $p<.001$, and baseline and 12-month follow-up (Mean Rank $=2.12), p<.001$. There was no significant difference between the $3-$ and $12-$ month follow-up mental health diagnoses $(p=.38)$.

To investigate whether substance use is related to a diagnosis of mental health Pearson's Chi-square tests were conducted (see Table 4). The chi square tests comparing mental health category and substance use status were significant at baseline, $\chi^{2}(2, N=794)=$ 18.02, $p<.001$, 3-month follow-up, $\chi^{2}(2, N=225)=15.23, p<.001$, and 12-month followup, $\chi^{2}(2, N=113)=18.20, p<.001$. At all three time points the proportion of participants who were languishing was significantly higher in the group who were still using substances $(26.3 \%, 14.4 \%, 15.3 \%)$ compared to those who were abstinent $(14.9 \%, 4.4 \%, 7.3 \%)$. Similarly, the proportion of those who were flourishing was significantly higher for those who were abstinent $(32.0 \%, 51.8 \%, 65.9 \%)$ compared to those who were still using $(19.1 \%$, $28.8 \%, 25.0 \%)$.

\section{[INSERT TABLE 4 HERE]}

\section{Continuous Mental Health}

To investigate continuous mental health, mixed-design ANOVA and ACL analyses were utilised. For the mixed-design ANOVA, reported substance use status (abstinent or using) at 3-month follow-up was used as the grouping variable (see Table 5). The results 
revealed a significant interaction between mental health and substance use status, $F(2,218)=$ 4.92, $p<.01$, partial $\eta^{2}=.04$. Figure 2 shows that participants started with a similar level of mental health. However, while mental health increases over time for both groups, abstinent individuals experience larger increases in mental health than those who use substances. There was an overall within-subjects effect of mental health across time, $F(2,218)=24.09, p<.01$, with significant differences between baseline and 3-month follow-up $(p<.001)$ and baseline and 12-month follow-up ( $p<.001)$, but not between the 3- and 12-month follow-ups $(p>.05)$. This indicates that improvements in mental health occur early in the recovery phase and as indicated in Figure 2, continue to improve for individuals who abstain and decrease slightly for individuals who use substances.

\section{[INSERT TABLE 5 HERE]}

Additionally, a significant main effect of substance use status was found, $F(1,109)=$ 13.60, $p<.001$. Congruent with Figure 2, simple effects indicated that mental health was not significantly different between abstainers and users at baseline $(p=.404)$. However, mental health was rated significantly higher by abstinent individuals than those who had used substances at 3-month $(p<.01)$, and 12-month follow-up $(p<.001)$.

\section{[INSERT FIGURE 2 HERE]}

Next, ACL models were applied; specifically, the antecedent, consequence, and reciprocal influence models. The ASI-Alcohol Severity, ASI-Drug use Severity and DAQ (cravings) measures were used as indicators of addiction problems. These three addiction measures were each used with the Mental Health Continuum (continuous variable) in three ACL analyses.

All models showed excellent fit, with Chi-square $(2)<5.5, p>.05$, Comparative fit index $>$.97, and Root Mean Square Error of Approximation <.05. Figure 3 presents the results. The variables tended to be moderately stable across time. There were no significant 
cross-lags going from mental health to substance use, providing no support for the mentalhealth as antecedent model. There were, however, significant cross-lags going from Time 2 measurement of substance use and cravings, and Time 3 mental health. These results support the mental health as consequence model. Time 2 and Time 3 measures represent residual change in this ACL model. Thus, if participants improved on the substance use variables from Time 1 to Time 2, they were more likely than those who did not improve to experience an increase in mental health from Time 2 to Time 3.

\section{[INSERT FIGURE 3 HERE]}

\section{Discussion}

This study examined whether Keyes' model of mental health had utility in the context of drug and alcohol misuse. Proportions of mental health diagnoses identified that flourishing, languishing and moderate mental health diagnoses were present in the current sample. Compared to American population estimates (16.9\%; Keyes, 2005), the current sample had higher levels of languishing at baseline (23.8\%) as might be expected. It was notable that following treatment at 3-month follow-up in the community, the frequency of those considered to be languishing was lower than in general population samples (Keyes, 2005). The rates of flourishing at baseline were similar between population and recovery samples (18\% and $21.9 \%$ respectively), however by the 12-month follow-up, the rates of flourishing were higher (39.8\%) and more comparable to those found among yoga practitioners (43.8\%; Ross, et al., 2013). There was a significant difference in mental health proportions between baseline and follow-up assessments, but not between the two follow-up assessment points.

There were associations between categorical mental health status and the use of substances. It was found that at post-treatment follow-ups, abstinence from substances was 
more commonly associated with flourishing mental health. Conversely, given that we cannot assume causality it may be that individuals with flourishing mental health may be more likely to maintain abstinence. The directional aspects of these relationships are discussed further when we review the results of the Autoregressive Cross-lag (ACL) analyses using continuous variables.

There was a significant interaction between substance use status (abstinent/used) and mental health over time. Those who were abstinent (based on the 3 month follow-up period) experienced significantly better mental health during the follow-up periods compared to those who continued to use. Participants who used substances achieved mean continuous mental health scores that were only either equal to, or marginally above, the mean baseline score of abstinent individuals. As a result of attrition and missing data, these findings are particularly relevant for those with more severe levels of alcohol misuse at treatment entry and may not generalise to those with less severe alcohol problems.

Finally, the model testing indicated that the data were most consistent with the mental health as a consequence model. That is, improved mental health appears to be a consequence of reduced severity of alcohol and other drug abuse. Similarly, better mental health follows reductions in cravings. The measures were administered at three time points; at entry to residential treatment; at 3-month follow-up after discharge and then 12 months postdischarge follow-up. Reductions in drug and alcohol severity and cravings appeared to precede improvements in mental health over these time periods. This result is consistent with the supposition that the receipt of treatment and the consolidation of treatment effects in the community lead to improved drug and alcohol outcomes, although without a control group we cannot conclude causation. However, the results do suggest that it is not just drug and alcohol specific outcomes, but also more general mental health that improve. 


\section{Study Implications}

Finding relatively low rates of languishing at follow-up compared to population rates, was somewhat surprising but may be partially explained by a contrast effect. That is, individuals who have experienced the challenges and hardships associated with substance abuse, which then have positive personal and emotional experiences through treatment, could respond disproportionately to such positive experiences. The contrast between their baseline levels of well-being and subsequent perceptions may lead more individuals to move out of the languishing range. This contrast effect would likely be further enhanced by the positive emotional reactivity found among individuals who are flourishing (Catalino \& Fredrickson, 2011). In addition, the relatively lower rates of languishing were found after participants had prioritised their well-being by receiving treatment. Similarly, the low rates of languishing amongst yoga practitioners was also attributed to the high priority they gave health and wellbeing (Ross, et al., 2013). However, the current frequencies and comparison may also be influenced by differences in rates of mental health between the US and Australian samples. This highlights the need to assess Australian community population rates of mental health as conceptualised by Keyes. Finally, some caution is needed in making comparisons between studies using either the short- or long-form of the Mental Health Continuum. The American population estimates of the mental health categories were generated from the long-form of the Mental Health Continuum, while the current and other studies, including the yoga practitioner study, have utilised the short-form. Although both forms of the Mental Health Continuum have demonstrated adequate validity and reliability (Keyes, 1998, 2002; Ryff \& Keyes, 1995), it is unclear whether they might result in variations of estimates.

The pre and post treatment differences in mental health diagnoses indicates that individuals may experience significant changes from treatment entry to post-discharge follow-up, but that these changes stabilise over the 12-months post-discharge. This pattern 
has been identified in previous research with stabilisation being a common goal in the early stages (less than 12-months) of recovery (Dennis, Scott, Funk, \& Foss, 2005; El-Guebaly, 2012; McLellan, Lewis, O'Brien, \& Kleber, 2000).

The majority of the sample experienced moderate mental health. While not mentally unhealthy, these individuals are not experiencing the benefits of flourishing (Keyes, 2005). The mental health of these individuals might be further increased, through enhancing or maintaining support resources. Such resources are broadly known as recovery capital (Cloud \& Granfield, 2008). Recovery capital includes variables such as spirituality, religion, life meaning, and 12-step affiliation (Laudet, Morgen, \& White, 2006) which are thought to reflect an individual's embeddedness in their social and cultural life (Granfield \& Cloud, 2001; Laudet, et al., 2006). Enhancing components of recovery capital may buffer the effects of stress (Laudet, et al., 2006; Laudet \& White, 2008) and lead to improved mental health. Future research is needed to confirm such relationships.

Languishing was found to be most common amongst individuals who had used substances. However, there were individual's at all three assessments who had remained abstinent and yet were languishing. This is the group that historically may have been referred to as “dry drunks” (Gogek, 1994). While abstinence has previously been utilised as a defintion of recovery (Garbutt, et al., 1999; Laudet \& White, 2010; Rudolf \& Watts, 2002), the current, and previous research highlights the limitations of using abstinence as an isolated outcome(Schwarzlose, et al., 2007). The inclusion of mental health indices is likely to more comprehensively capture the experience of individuals in recovery (Laudet, 2007).

Finally, evidence for the consequential model suggests that by improving drug and alcohol outcomes other mental health indicators also improve. The study is not able to clarify the mechanisms by which this occurs, but the provision of treatment over the study period suggests this as a potential if not likely mechanism. The Salvation Army residential treatment 
program is faith-based and subscribes to a 12-step treatment model in what is described as a modified therapeutic community. However, there are multiple other components such as individual counselling, family support and work opportunities provided (e.g., gardening, food services). There is a need for future research to try to provide evidence for the most helpful ingredients in treatment that maximise both substance abuse and mental health outcomes.

\section{Limitations and Future Directions}

Limitations of the study include reduced sample sizes for follow-up periods, use of only faith-based treatment programs, and the primarily male sample (79.9\%). Despite attrition analyses suggesting only alcohol addiction severity may be different for those who completed all follow-ups and those who did not, it is still possible there were other unexplored variables that could potentially bias the findings. Although the ASI is a widely used measure of substance use (McLellan, et al., 1992), it is a self-report measure. Future research may benefit from including objective measures of substance use.

There are many reasons for failing to retain participants at follow-up with the more common being: re-entry into recovery services; not providing consent to be contacted at follow-up; incorrect contact details; and withdrawing consent at follow-up contact. Future research would benefit from utilising balanced gender and a combination of secular and nonsecular treatment comparison groups. In addition, the majority of previous literature has arisen from North American culture (Dennis, et al., 2005), whereas the current study utilises an Australian sample. While this broadens research on mental health in addictions contexts, it would be useful to obtain Australian community normative data on the mental health continuum to provide an additional point of reference to quantify rates of mental health recovery.

Despite these limitations, this study offers a unique insight into how mental health relates to recovery from substance misuse. While the comorbidity of mental illness and 
substance misuse has previously been investigated, this is one of the first studies to document the prevalence of mental health diagnoses. 


\section{References}

Arbuckle, J. (2012). Amos 21. Spring House, PA: Amos Development Corporation.

Best, D., Gow, J., Knox, T., Taylor, A., Groshkova, T., \& White, W. (2012). Mapping the recovery stories of drinkers and drug users in Glasgow: Quality of life and its associations with measures of recovery capital. Drug and Alcohol Review, 31(3), 334341. doi: 10.1111/j.1465-3362.2011.00321.x

Bowersox, N. W., Saunders, S. M., \& Wojcik, J. V. (2009). An evaluation of the utility of statistical versus clinical significance in determining improvement in alcohol and other drug (AOD) treatment in correctional settings. Alcoholism Treatment Quarterly, 27(1), 113-129. doi: 10.1080/07347320802591700

Catalino, L. I., \& Fredrickson, B. L. (2011). A Tuesday in the life of a flourisher: the role of positive emotional reactivity in optimal mental health. Emotion, 11(4), 938-950. doi: 10.1037/a0024889

Cloud, W., \& Granfield, R. (2008). Conceptualizing recovery capital: Expansion of a theoretical construct. Substance Use \& Misuse, 43(12-13), 1971-1971. doi: $10.1080 / 10826080802289762$

Coombs, T., \& Meehan, T. (2003). Mental health outcomes in Australia: Issues for mental health nurses. International Journal of Mental Health Nursing, 12(3), 163-164. doi: 10.1046/j.1440-0979.2003.00285.x

Cooper, M. L., Frone, M. R., Russell, M., \& Mudar, P. (1995). Drinking to regulate positive and negative emotions: A motivational model of alcohol use. Journal of Personality and Social Psychology, 69(5), 990-1005.

De Maeyer, J., Vanderplasschen, W., Lammertyn, J., van Nieuwenhuizen, C., \& Broekaert, E. (2011). Exploratory study on domain-specific determinants of opiate-dependent 
individuals' quality of life. European Addiction Research, 17(4), 198-210. doi: $10.1159 / 000324353$

Deane, F. P., Kelly, P. J., Crowe, T. P., Lyons, G. C. B., \& Cridland, E. K. (2014). The feasibility of telephone follow-up interviews for monitoring treatment outcomes of Australian residential drug and alcohol treatment programs. Substance Abuse, 35(1), 21-29. doi: 10.1080/08897077.2013.789815

Dennis, M. L., Scott, C. K., Funk, R., \& Foss, M. A. (2005). The duration and correlates of addiction and treatment careers. Journal of Substance Abuse Treatment, 28(2), S51S62. doi: 10.1016/j.jsat.2004.10.013

Donovan, D., Mattson, M. E., Cisler, R. A., Longabaugh, R., \& Zweben, A. (2005). Quality of life as an outcome measure in alcoholism treatment research. Journal of Studies on Alcohol and Drugs, Supplement(15), 119-139.

El-Guebaly, N. (2012). The meanings of recovery from addiction: Evolution and promises. Journal of Addiction Medicine, 6(1), 1.

Enders, C. K., \& Bandalos, D. L. (2001). The relative performance of full information maximum liklihood estimation for missing data in structural equation models. Structural Equation Modeling, 8, 430-457.

Flaherty, J. A., McGuire, H. T., \& Gatski, R. L. (1955). The psychodynamics of the dry drunk. The American Journal of Psychiatry, 112(6), 460.

Garbutt, J. C., West, S. L., Carey, T. S., Lohr, K. N., \& Crews, F. T. (1999). Pharmacological treatment of alcohol dependence: A review of the evidence. Journal of the American Medical Association, 281(14), 1318-1325. doi: 10.1001/jama.281.14.1318

Gogek, E. B. (1994). The dry drunk syndrome: Subtype of depression? The American Journal of Psychiatry, 151(6), 947-948. 
Granfield, R., \& Cloud, W. (2001). Social context and "natural recovery": the role of social capital in the resolution of drug-associated problems. Substance Use \& Misuse, 36(11), 1543.

Howell, D. C. (2008). The analysis of missing data. In W. Outhwaite \& S. Turner (Eds.), Handbook of social science methodology (pp. 208-224). London: Sage.

Keyes, C. L. M. (1998). Social well-being. Social Psychology Quarterly, 61(2), 121-140. doi: $10.2307 / 2787065$

Keyes, C. L. M. (2002). The mental health continuum: From languishing to flourishing in life. Journal of Health and Social Behavior, 43(2), 207-222.

Keyes, C. L. M. (2005). Mental illness and/or mental health? Investigating axioms of the complete state model of health. Journal of Consulting and Clinical Psychology, 73(3), 539-548. doi: 10.1037/0022-006x.73.3.539

Keyes, C. L. M. (2007). Promoting and protecting mental health as flourishing: A complementary strategy for improving national mental health. American Psychologist, 62(2), 95-108. doi: 10.1037/0003-066x.62.2.95

Keyes, C. L. M. (2009). Brief description of the mental health continuum short form (MHCSF) Retrieved 10 May, 2012, from http://www.sociology.emory.edu/ckeyes/

Keyes, C. L. M., \& Simoes, E. J. (2012). To flourish or not: Positive mental health and allcause mortality. American Journal of Public Health, 102(11), 2164-2172. doi: 10.2105/ajph.2012.300918

Keyes, C. L. M., \& Westerhof, G. J. (2012). Chronological and subjective age differences in flourishing mental health and major depressive episode. Aging \& Mental Health 16(1), 67-74. 
Khantzian, E. J. (1997). The self-medication hypothesis of substance use disorders: A reconsideration and recent applications. Harvard Review of Psychiatry, 4(5), 231-244. doi: $10.3109 / 10673229709030550$

Laudet, A. (2007). What does recovery mean to you? Lessons from the recovery experience for research and practice. Journal of Substance Abuse Treatment, 33(3), 243-256. doi: 10.1016/j.jsat.2007.04.014

Laudet, A., Cleland, C. M., Magura, S., Vogel, H. S., \& Knight, E. L. (2004). Social support mediates the effects of dual-focus mutual aid groups on abstinence from substance use. American Journal of Community Psychology, 34(3-4), 175-185. doi: 10.1007/s10464-004-7413-5

Laudet, A., \& Humphreys, K. (2013). Promoting recovery in an evolving policy context: What do we know and what do we need to know about recovery support services? Journal of Substance Abuse Treatment, 45(1), 126-133. doi: 10.1016/j.jsat.2013.01.009

Laudet, A., Morgen, K., \& White, W. (2006). The role of social supports, spirituality, religiousness, life meaning and affiliation with 12-step fellowships in quality of life datisfaction among individuals in recovery from alcohol and drug problems. Alcoholism Treatment Quarterly, 24(1), 33-73. doi: 10.1300/J020v24n01_04

Laudet, A., \& White, W. (2008). Recovery capital as prospective predictor of sustained recovery, life satisfaction, and stress among former poly-substance users. Substance Use \& Misuse, 43(1), 27-27. doi: 10.1080/10826080701681473

Laudet, A., \& White, W. (2010). What are your priorities right now? Identifying service needs across recovery stages to inform service development. Journal of Substance Abuse Treatment, 38(1), 51-59. doi: 10.1016/j.jsat.2009.06.003 
Lovibond, S. H., \& Lovibond, P. F. (1995). Manual for the depression anxiety stress scales (2nd ed.). Sydney, NSW: Psychology Foundation of Australia.

Marshall, S., Parker, P., Ciarrochi, J., \& Heaven, P. (2014). Is self-esteem a cause or consequence of social support: A five year longitudinal study. Child Development, 85(1275-1291).

McLellan, A. T., Kushner, H., Metzger, D., Peters, R., \& et al. (1992). The fifth edition of the Addiction Severity Index. Journal of Substance Abuse Treatment, 9(3), 199.

McLellan, A. T., Lewis, D. C., O'Brien, C. P., \& Kleber, H. D. (2000). Drug dependence, a chronic medical illness: Implications for treatment, insurance, and outcomes evaluation. The Journal of the American Medical Association, 284(13), 1689-1695. doi: 10.1001/jama.284.13.1689

Mo, C., Deane, F. P., Lyons, G. C. B., \& Kelly, P. J. (2013). Factor analysis and validity of a short six-item version of the Desires for Alcohol Questionnaire. Journal of Substance Abuse Treatment, 44(5), 557-564. doi: 10.1016/j.jsat.2012.12.002

Mortlock, K. S., Deane, F. P., \& Crowe, T. P. (2011). Screening for mental disorder comorbidity in Australian alcohol and other drug residential treatment settings. Journal of Substance Abuse Treatment, 40(4), 397-404. doi: 10.1016/j.jsat.2011.01.002

Provencher, H. L., \& Keyes, C. L. M. (2011). Complete mental health recovery: Bridging mental illness with positive mental health. Journal of Public Mental Health, 10(1), 57.

Ross, A., Friedmann, E., Bevans, M., \& Thomas, S. (2013). National survey of yoga practitioners: Mental and physical health benefits. Complementary Therapies in Medicine, 21(4), 313-323. doi: 10.1016/j.ctim.2013.04.001 
Rudolf, H., \& Watts, J. (2002). Quality of life in substance abuse and dependency. International Review of Psychiatry, 14(3), 190-197. doi: $10.1080 / 09540260220144975$

Ryff, C. D., \& Keyes, C. L. M. (1995). The structure of psychological well-being revisited. Journal of Personality and Social Psychology, 69(4), 719-727. doi: 10.1037/00223514.69.4.719

Scheier, M. F., Wrosch, C., Baum, A., Cohen, S., Martire, L. M., Matthews, K. A., . . . Zdaniuk, B. (2006). The Life Engagement Test: Assessing purpose in life. Journal of Behavioral Medicine, 29(3), 291-298. doi: 10.1007/s10865-005-9044-1

Schwarzlose, J., Belleau, C., DuPont, R. L., Erickson, C. K., Flaherty, M. T., Galanter, M., . . . Betty Ford Institute Consensus, P. (2007). What is recovery? A working definition from the Betty Ford Institute. Journal of Substance Abuse Treatment, 33(3), 221-228. doi: 10.1016/j.jsat.2007.06.001

Sklar, S. M., \& Turner, N. E. (1999). A brief measure for the assessment of coping selfefficacy among alcohol and other drug users. Addiction, 94(5), 723-723. doi: 10.1046/j.1360-0443.1999.94572310.x

Substance Abuse \& Mental Health Services Administration. (2011). SAMHSA announces a working definition of "recovery" from mental disorders and substance use disorders Retrieved 18/01, 2012, from http://www.samhsa.gov/newsroom/advisories/1112223420.aspx

Tabachnick, B. G., \& Fidell, L. S. (2013). Using multivariate statistics. Boston: Pearson Education.

Zanis, D. A., McLellan, A. T., \& Corse, S. (1997). Is the Addiction Severity Index a reliable and valid assessment instrument among clients with severe and persistent mental 
illness and substance abuse disorders? Community Mental Health Journal, 33(3), 213227. doi: 10.1023/a:1025085310814 
Figure Captions:

Figure 1. Participant flow over baseline and follow-up assessments.

Figure 2. Changes in continuous mental health scores between assessment periods as a function of substance use status $(n=111)$.

Figure 3. Longitudinal relationships of mental health with alcohol and substance use, and cravings. Note. The three standardized coefficients represent the relationships for substances, alcohol use, and cravings, respectively. Two-year auto correlations were estimated but are not presented in the figure. Bolded coefficients are significant at $p<.05,{ }^{*} p=.055$. 